\title{
Imaging diagnostics for transitional discs
}

\author{
M. de Juan Ovelar ${ }^{1}$, M. Min², C. Dominik ${ }^{2,3}$, C. Thalmann² ${ }^{2}$, P. Pinilla ${ }^{4}$, M. Benisty ${ }^{5}$, and T. Birnstiel ${ }^{6}$ \\ ${ }^{1}$ Leiden Observatory, Leiden University, PO Box 9513, 2300RA Leiden, The Netherlands \\ e-mail: mjovelar@strw. leidenuniv.nl \\ 2 Astronomical Institute Anton Pannekoek, University of Amsterdam, 1090 GE Amsterdam, The Netherlands \\ 3 Department of Astrophysics/IMAPP, Radboud University Nijmegen, 6500 GL Nijmegen, The Netherlands \\ 4 Institut für Theoretische Astrophysik, Universität Heidelberg, Albert-Ueberle-Straße 2, 69120 Heidelberg, Germany \\ 5 Institut de Planétologie et Astrophysique Grenoble, 414 rue de la Piscine, 38400 St-Martin d'Hères, France \\ ${ }^{6}$ Harvard-Smithsonian Center for Astrophysics, 60 Garden Street, Cambridge, MA 02138, USA
}

Received 5 July 2013 / Accepted 31 August 2013

\section{ABSTRACT}

\begin{abstract}
Transitional discs are a special type of protoplanetary disc, where planet formation is thought to be taking place. These objects feature characteristic inner cavities and/or gaps of a few tens of AUs in sub-millimetre images of the disc. This signature suggests a localised depletion of matter in the disc that could be caused by planet formation processes. However, recent observations have revealed differences in the structures imaged at different wavelengths in some of these discs. In this paper, we aim to explain these observational differences using self-consistent physical 2D hydrodynamical and dust evolution models of these objects, assuming their morphology is indeed generated by the presence of a planet. We use these models to derive the distribution of gas and dust in a theoretical planet-hosting disc for various planet masses and orbital separations. We then simulate observations of the emitted and scattered light from these models with Very Large Telescope (VLT)/SPHERE-ZIMPOL, Subaru/HiCIAO, VLT/VISIR, and ALMA. We do this by first computing the full resolution images of the models at different wavelengths and then simulating the observations while accounting for the characteristics of each particular instrument. The presence of the planet generates pressure bumps in the gas distribution of the disc, whose characteristics strongly depend on the planet mass and position. These bumps cause large grains to accumulate, while small grains are allowed into inner regions. This spatial differentiation of the grain sizes explains the differences in the observations, since different wavelengths and observing techniques trace different parts of the dust size distribution. Based on this effect, we conclude that the combination of visible/near-infrared polarimetric and sub-mm images is the best strategy to constrain the properties of the unseen planet responsible for the disc structure.
\end{abstract}

Key words. protoplanetary disks - planet-disk interactions - circumstellar matter - methods: observational

\section{Introduction}

Transitional discs are generally believed to be the result of a planet-forming stage in a circumstellar disc. As such, the study of these objects provides important information that can help us to constrain the physics of the planet-formation process. Therefore, a considerable amount of effort is currently being invested to theoretically and observationally understand these objects better. In general, the spectral energy distribution (SED) of these sources and interferometric measurements at submillimitre wavelengths show evidence of inner cavities and/or gaps (e.g. Strom et al. 1989; Espaillat et al. 2010; Andrews et al. 2011). One of the interpretations of these observations is that a fraction of the material in the disc is depleted by a forming planet (See Williams \& Cieza 2011, for an extensive review).

Recent observational work has called this conclusion into question based on measurements of the accretion rate (e.g. Calvet et al. 2005; Espaillat et al. 2007) and polarimetric observations of the inner regions of the disc (Dong et al. 2012). The latter, carried out as a part of the near-infrared (NIR) Strategic Explorations of Exoplanets and Disks with Subaru survey (SEEDS, Tamura 2009), is of particular interest since it involves direct imaging of the disc structure. The results of the survey were surprising with regard to a number of transition discs classified as such based on sub-mm emission observations. The polarimetric images of these discs did not show the expected gap
(Dong et al. 2012). A parametric model of the disc with a continuous radial distribution of small grains $(\sim 1 \mu \mathrm{m})$ and a significant depletion of big grains $(\sim 1 \mathrm{~mm})$ in the inner regions of the disc was found to reproduce these observational discrepancies. These regions would appear empty in the sub-mm images whereas the presence of small grains that scatter light very efficiently in the NIR wavelengths could explain the polarimetric measurements. However the Dong et al. (2012) study could not provide an explanation of the physical mechanisms causing this differentiation of big and small dust grain distributions across the radial extent of the disc due to the parametric nature of the model. Instead, it is merely suggested that some filtering mechanism must be active.

Theoretical studies, such as Rice et al. (2006); Zhu et al. (2011, 2012); Pinilla et al. (2012a), provide a potential physical explanation. The main drive for these studies is to explain how particles of dust in a disc can grow up to large sizes $(\sim 1 \mathrm{~m})$ without being dragged towards the star by the radial drift mechanism. This constitutes one of the long-standing problems in planet formation, known as the "1-m barrier" problem (Weidenschilling 1977; Brauer et al. 2008). In particular, in one of the latest studies (Pinilla et al. 2012a) explored the influence the presence of a planet has on the distribution of gas and dust in the disc. In this study, the authors combine 2D hydrodynamical simulations of the evolution of the gas distribution in a disc that hosts a planet with state-of-the-art dust evolution models. For the first time, 
these models include self-consistent calculations of the radial drift, coagulation, and fragmentation mechanisms undergone by the dust (Birnstiel et al. 2010). They showed that the presence of a planet generates pressure gradients in the gas distribution that cause the velocity of the gas to increase to near-Keplerian values at specific radial positions. This reduces the differential velocity between dust and gas particles and, therefore, the drag force exerted on the dust. As a consequence, large particles $(\sim 1 \mathrm{~mm})$, which are less affected by the gas drag, accumulate in those radial locations where the pressure reaches a maximum, which allows them to grow protected from the radial drift. Smaller grains, which are still coupled to the gas, follow the accretion flow into the inner regions of the disc. The trapping and filtering characteristics and the radial position of these bumps are highly dependent on the mass and position of the planet.

If these models indeed reproduce the physical processes taking place in a transitional disc, it may be possible to constrain characteristics of the unseen planet by studying the sizedifferentiated dust distribution generated by its presence. The observational differences found between sub-mm emission and NIR scattered images then become a powerful diagnostic tool for transitional discs and the planets they host. In this work, we perform a theoretical study simulating observations of hydrodynamical and dust evolution models, which are similar to those presented in Pinilla et al. (2012a) with the Very Large Telescope (VLT) Spectro-Polarimetric High-contrast Exoplanet REsearch instrument (SPHERE) and its polarimeter ZIMPOL (Zurich Imaging Polarimeter), the High Contrast Instrument for the Subaru Next Generation Adaptive Optics (HiCIAO), the VLT spectrometer and imager for the mid-infrared (VISIR), and the Atacama Large Millimeter/submillimeter Array (ALMA). Our aim is to 1) test whether these models can reproduce the general characteristic features found in observations; 2) analyse what different imaging techniques can tell us about the dust distribution; and 3) detect the best imaging strategy to constrain characteristics of the planet from dust measurements, such as mass and position. We would like to clarify that we do not aim to explain detailed characteristics revealed by particular observations of transitional discs.

The study is organised as follows: in Sect. 2, we describe the methodology used to obtain the disc models and the simulated observations. In Sect. 3 we present images and radial profiles obtained for the different cases and instruments considered and the discussion of those results in Sect. 4. Finally, we provide a short summary of the study and list our conclusions in Sect. 5.

\section{Method}

To generate images of a transitional disc, we base our study in the disc-planet interaction models presented in Pinilla et al. (2012a). These models combine two-dimensional hydrodynamical and dust evolution simulations, including radial drift, coagulation, and fragmentation, to self-consistently reproduce the evolution of the gas and dust in the disc. We consider the cases of a disc with a planet of masses $M_{\mathrm{p}}=[1,9,15] M_{\mathrm{Jup}}$ at radial positions $R_{\mathrm{p}}=[20,40,60] \mathrm{AU}$. We take the resulting distribution of dust and gas for these three cases after $3 \mathrm{Myr}$ of evolution and input them in the Monte-Carlo radiative transfer code MCMax to produce full-resolution intensity and polarised-intensity images of the emitted and scattered flux. Finally, we simulate realistic observations with VLT/SPHERE ZIMPOL, Subaru/HiCIAO, VLT/VISIR and ALMA by either using specific instrument simulators or convolving the full resolution images with the characteristic point spread function (PSF) and adding realistic effects,
Table 1. Disc-planet simulation input parameters.

\begin{tabular}{ll}
\hline \hline Temperature of the star $\left(T_{\text {star }}\right)$ & $4730 \mathrm{~K}$ \\
Radius of the star $\left(R_{\text {star }}\right)$ & $1.7 M_{\odot}$ \\
Mass of the star $\left(M_{\text {star }}\right)$ & $1 M_{\odot}$ \\
Mass of the disc $\left(M_{\text {disc }}\right)$ & $0.0525 M_{\odot}$ \\
Position of the planet $\left(R_{\mathrm{p}}\right)$ & {$[20,40,60] \mathrm{AU}$} \\
Fragmentation velocity $\left(v_{\mathrm{f}}\right)$ & $10 \mathrm{~m} / \mathrm{s}$ \\
Inner disc radius $\left(R_{\text {disc,inn }}\right)$ & $0.025 R_{\mathrm{p}}$ \\
Outer disc radius $\left(R_{\text {disc,out }}\right)$ & $7.0 R_{\mathrm{p}}$ \\
Solid density of dust particles $\left(\rho_{\text {dust }}\right)$ & $1.2 \mathrm{~g} / \mathrm{cm}^{3}$ \\
Alpha viscosity $(\alpha)$ & $10^{-3}$ \\
\hline
\end{tabular}

such as noise, and decrease in resolution due to seeing. In the following subsections, we present the details of the steps followed.

\subsection{Disc-planet interaction models}

To obtain the gas and dust distribution of our transitional discs, we follow the same simulation procedure used in Pinilla et al. (2012a). The results are obtained by first solving the equations for the hydrodynamical interactions between the gas in the disc and the planet and then computing the evolution of the dust in a disc with the obtained gas surface distribution. The first computation is done by means of the two-dimensional hydrodynamical code FARGO (Masset 2000), which uses finite differences to solve the Navier-Stokes and continuity equations in a grid of annular cells that define the disc. The simulations were computed selecting open boundary conditions to allow the material to leave the grid. The logarithmically extended radial grid is taken for each case from $R_{\text {disc,inn }}=0.025$ to $R_{\text {disc,out }}=7.0$ using normalised units, such that the planet is located at $R_{\mathrm{p}}=1.0$. Table 1 lists the input parameters used in these simulations. They are the same as in Pinilla et al. (2012a) but considering $\Sigma \propto r^{-1}$, a kinematic viscosity of $v=\alpha c_{\mathrm{s}} h$ with $\alpha=10^{-3}$, and normalising the mass of the disc to $M_{\text {disc }}=0.0525 M_{\odot}$. Stellar parameters are those typical of T-Tauri stars. Note that parameters defining the geometry of the disc, $R_{\text {disc,inn }}$ and $R_{\text {disc,out }}$, are defined with respect to the position of the planet such that they are always far enough from it to allow for an appropriate study of its effects in the inner and outer discs. Although this link may not be necessarily physical, it allows for comparison of the different planet separation cases without increasing the radial resolution of the simulations, and therefore the required computational time.

The hydrodynamical simulations are done until the disc reaches a quasi-stable state after $\sim 1000$ planet orbits. The 2D gas surface density is then azimuthally averaged and used as the initial condition for the dust evolution modelling. During the dust evolution modeling, we note that the gas surface density remains constant on timescales of millions of years, hence any mechanism, such as photoevaporation, that may disturb the gas density is omitted. For the dust evolution simulations, we use the 1D code described in Birnstiel et al. (2010), which computes the growth and fragmentation that happens in the radial dust distribution due to radial drift, turbulent mixing, and gas drag forces. The dust is initially distributed such that the dust-to-gas ratio is $1 \%$ with an initial size of $1 \mu \mathrm{m}$. The dust distribution is evolved for $3 \mathrm{Myr}$. We follow this process for all planet mass and radii cases, where $M_{\mathrm{p}}=[1,9,15] M_{\mathrm{Jup}}$ and $R_{\mathrm{p}}=[20,40,60] \mathrm{AU}$.

Figure 1 shows the results of these simulations for each planet mass studied and for the specific case of $R_{\mathrm{p}}=20 \mathrm{AU}$. Top panels show the 2D gas surface density after 1000 orbits of evolution. For each case, it is clear how the shape of the gap 

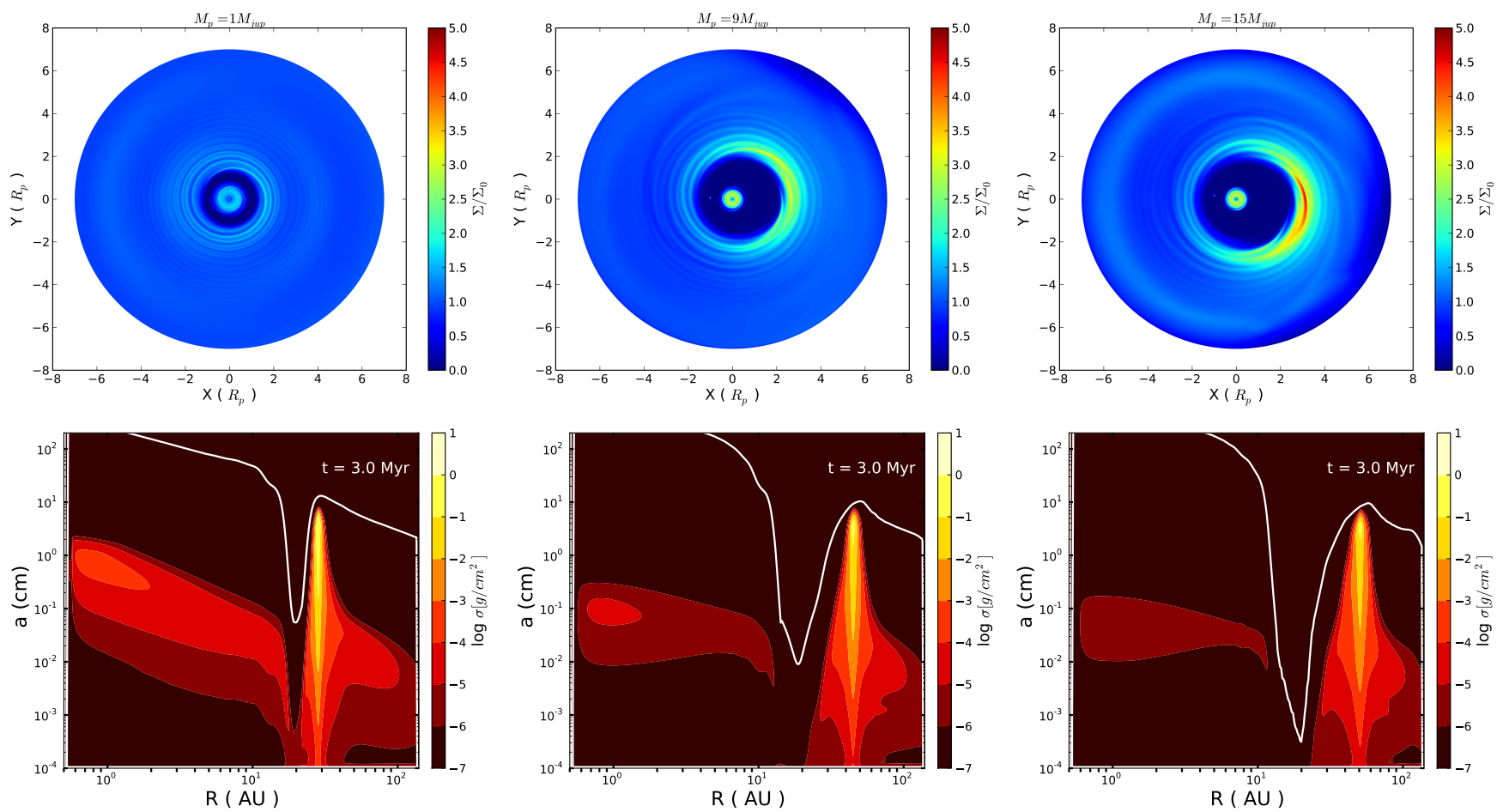

Fig. 1. Top panels: 2D gas surface density for $[1,9,15] M_{\text {jup }}$ after 1000 orbits of evolution. Bottom panels: vertically integrated dust-density distribution for each planet mass and for the case of $R_{\mathrm{p}}=20 \mathrm{AU}$. The white line corresponds to the size of particles that feel the highest radial drift and is proportional to the gas surface density.

varies with the mass of the planet. For the case of $15 M_{\text {jup }}$, a vortex even appears at the outer edge of the gap due to the high mass of the planet (Ataiee et al. 2013).

Bottom panels of Fig. 1 show the vertically integrated dust density distribution (see Pinilla et al. 2012a, Eqs. (5) and (6)). The solid white line indicates the size of particles that feel the highest radial drift, which is directly proportional to the azimuthally-averaged gas surface density (see Pinilla et al. 2012a, for details). The presence of the planet clearly perturbs the gas and dust density distributions, dividing the disc into inner and outer regions, where the distribution of dust particle sizes is clearly different. Due to the 1D nature of the dust evolution code, we note that the dust distribution cannot reproduce the asymmetries caused by the presence of e.g. vortices in the gas distribution. For the purposes of our study, where the aim is to analyse the radial morphology of the dust distribution, this approximation is sufficient.

\subsection{Radiative transfer simulations}

To compute the emitted and scattered flux of the disc, we use the Monte-Carlo radiative transfer code MCMax (Min et al. 2009). Given the size and composition of the particles and the characteristics of the central star, MCMax self-consistently solves the temperature and vertical structure of the distribution of gas and dust in the disc including the effect of dust settling. It produces common observables for the study of the disc, such as SEDs or emission and scattering images, at the desired wavelengths and inclination angles. The code is widely used to compare theoretical models of discs with observations (see Mulders et al. 2010, 2011; Mulders \& Dominik 2012; de Vries et al. 2012; Canovas et al. 2012; Jeffers et al. 2012; Lombaert et al. 2012; Honda et al. 2012; Min et al. 2012; Mulders et al. 2013, for some examples).
The MCMax program reads the distribution of gas and dust obtained from the disc-planet models for all planet mass and separation cases and also reads the central star and general disc parameters. It requires a composition of the dust to compute the opacities and temperatures. We model this composition as a mixture of silicates $(\sim 58 \%)$, iron sulphide $(\sim 18 \%)$, and carbonaceous dust grains $(\sim 24 \%)$ with an average density of $\rho_{\text {mix }}=$ $3.2 \mathrm{~g} / \mathrm{cm}^{3}$ (Min et al. 2011). We set a porosity for the dust grains of $p=0.625$, which corresponds to the density $\rho=1.2 \mathrm{~g} / \mathrm{cm}^{3}$ used in the dust evolution simulations. The indices of refraction needed to compute the opacities are obtained from the following: Dorschner et al. (1995); Henning \& Stognienko (1996) for the silicates, Begemann et al. (1994) for the iron sulphide, and Preibisch et al. (1993) for the carbonaceous dust grains. Provided a value for the viscous turbulence $(\alpha)$, MCMax selfconsistently simulates the settling effect. We set this value to that of the disc-planet simulations (i.e. $\alpha=10^{-3}$ ). The vertical structure of the gas in the disc is solved iteratively under the assumption of vertical hydrostatic equilibrium. The vertical structure of the dust is then computed using settling and vertical turbulent mixing.

With the resultant temperature and vertical structure, MCMax produces theoretical intensity and polarised-intensity images of the disc at the desired wavelength. In this paper, we discuss the results obtained for wavelengths of $\lambda=$ $[0.65,1.6,20,850] \mu \mathrm{m}$, which are the most commonly used for imaging diagnostics of circumstellar matter.

\subsection{Simulated observations}

To simulate realistic observations of the modelled discs, we select a set of currently available (Subaru/HiCIAO and VLT/VISIR) and near-future instruments (VLT/SPHEREZIMPOL and ALMA Complete Array) that are (or are likely 
Table 2. Wavelength and resolution of the simulated images.

\begin{tabular}{lccc}
\hline \hline Instrument & $1.2 \lambda / D$ & Final & $d=140 \mathrm{pc}$ \\
\hline SPHERE ZIMPOL & $0.02^{\prime \prime}$ & $0.03^{\prime \prime}$ & $\sim 4 \mathrm{AU}$ \\
Subaru HiCIAO & $0.04^{\prime \prime}$ & $0.09^{\prime \prime}$ & $\sim 13 \mathrm{AU}$ \\
VLT VISIR & $0.6^{\prime \prime}$ & $0.62^{\prime \prime}$ & $\sim 87 \mathrm{AU}$ \\
ALMA complete & $0.013^{\prime \prime}$ & $0.015^{\prime \prime}$ & $\sim 2 \mathrm{AU}$ \\
\hline
\end{tabular}

to be) leading the field of imaging circumstellar environments. These instruments work in different regions of the electromagnetic spectrum, thus probing different features in the disc. We select a filter from the ones available in each instrument $(R I$, $H, Q$, and $850 \mu \mathrm{m}$ ) and produce an MCMax full-resolution image for a particular wavelength in the filter range $(\lambda=$ $[0.65,1.6,20,850] \mu \mathrm{m}$ respectively). Then we either process these images using a specific instrument simulator or convolving them with a measured or simulated PSF of the instrument (depending on the case), and adding photon noise and loss of resolution accounting for realistic observational effects. We assume an exposure time of $t_{\mathrm{obs}}=1200 \mathrm{~s}$ with all instruments. Table 2 shows the the theoretical and final resolution obtained for the simulated observation with each instrument. In the following paragraphs, we explain the details of the image processing followed in each case.

- R-band intensity and polarised-intensity images with SPHERE ZIMPOL

Intensity and polarised-intensity observations with SPHERE in the $R$-band are simulated with the SPHERE ZIMPOL simulator that comes as part of the SPHERE software package (Thalmann et al. 2008). The SPHERE instrument (Beuzit et al. 2006) is a planet finder designed for the VLT which is planned to see first light by the end of 2013. By means of its polarimeter ZIMPOL, it provides linear polarimetric imaging capabilities for the characterisation of circumstellar environments and exoplanets (Gisler et al. 2004; Stuik et al. 2005; Thalmann et al. 2008; Roelfsema et al. 2010; Schmid et al. 2010). The simulator takes fullresolution intensity and polarimetric images as inputs and generates the observed images by simulating the artifacts and aberrations caused by the optical system. We choose the $R I$ filter in the simulator and process the MCMax intensity, Stokes $Q$, and $U$ images. The final polarised-intensity image is obtained as $P I=\sqrt{Q^{2}+U^{2}}$.

\section{- H-band intensity and polarised-intensity images with} $\mathrm{HiCIAO}$

In the case of $H$-band images, we convolve the full resolution intensity and Stokes $Q$ and $U$ images with a measured HiCIAO $H$-band PSF. This PSF is obtained from the publicly available ACORNS-ADI data reduction pipeline (Brandt et al. 2013). We add photon noise to the convolved images as follows. First, we add photon noise to the convolved intensity image. Then, the previous noise-free intensity image is subtracted from this to generate a photon noise map. We do this two consecutive times to generate different maps for the $Q$ and $U$ images. Each map is divided by two, assuming that half of the observing time goes to each linear polarisation measurement (i.e. $Q$ and $U$ ), and the result is added to the convolved $Q$ and $U$ images. The final polarised-intensity image is then computed again as $P I=\sqrt{Q^{2}+U^{2}}$. The final resolution obtained in this case is determined by the full width half maximum (FWHM) of the measured PSF.

\section{- Q-band intensity images with VISIR}

To simulate $Q$-band observations, we use MCMax. Provided the dimensions of the primary and secondary mirrors and the exposure time, the code can generate a theoretical PSF to be convolved with the images and compute the corresponding photon noise. There is also the option to specify an angular width to be added to the simulated resolution determined by the PSF. This accounts for the effect of the observing conditions (i.e., seeing). We specify values for the primary mirror of $D_{1}=8.4 \mathrm{~m}$, secondary mirror of and $D_{2}=1 \mathrm{~m}$, an exposure time of $t_{\mathrm{obs}}=12000 \mathrm{~s}$ and a seeing width of $w=0.05^{\prime \prime}$.

\section{- $850 \mu \mathrm{m}$ intensity images with ALMA}

The ALMA observations are simulated in a simplified way. The spatial resolution of ALMA can be estimated by calculating the resolution of a telescope with a primary mirror diameter as big as the baseline of the antenna array. For our study, we assume the maximum baseline provided by ALMA complete, which corresponds to $16 \mathrm{~km}$, and the diffraction-limited resolution at $850 \mu \mathrm{m}$, which is 13 mas. The ALMA complete array was assembled and inaugurated in March 2013, although some antennas are still being tested. Observations for the next cycle (Early Science-Cycle 2) with still reduced capabilities are expected to be performed in mid-2014. Full operations are expected to follow shortly after the end of Cycle 2 operations. To allow for slightly reduced performance, we use 15 mas as the FWHM of the PSF. We adjust the exposure time to account for the fact that the actual collecting area of ALMA is less than that of a telescope with a mirror as big as the baseline. In the final configuration of a completed ALMA the collecting area is provided by 50 antennas of diameter $D=12 \mathrm{~m}$ (we use only the $12 \mathrm{~m}$ antennas). Therefore, we reduce the effective observing time $\left(t_{\mathrm{obs}}=12000 \mathrm{~s}\right)$ by a factor of $f_{\text {corr. }}=\left(50 \times 6^{2}\right) / 8000^{2}=2.81 \times 10^{-5}$. We then fold the theoretical image with the final PSF of 15 mas to produce the images presented here.

\section{Results}

Figure 2 shows a selection of the obtained simulated observations for all planet mass cases at $R_{\mathrm{p}}=20 \mathrm{AU}$ and after $3 \mathrm{Myr}$ of evolution. From left to right, columns in the figure show polarised-intensity (PI) for $R$-band with ZIMPOL, $H$-band PI with HiCIAO, and intensity $(I)$ with ALMA at $850 \mu \mathrm{m}$, respectively. Rows show the different planet mass cases (i.e., $M_{\mathrm{p}}=$ $[1,9,15] M_{\text {Jup }}$ from top to bottom, respectively), and the white dot represents the position of the planet at $20 \mathrm{AU}$. Unless otherwise noted, radii lower than $R<R_{\mathrm{p}}$ or larger than $R>R_{\mathrm{p}}$ are referred to as inner and outer regions of the disc, respectively.

The polarised-intensity images obtained with the polarimetric capabilities of ZIMPOL (first column) reveal the structure of the disc remarkably well. In all planet mass cases, a gap at the position of the planet is detectable. Moreover, the three cases are different because the inner regions are actually resolved. In the case of polarimetric images obtained with HiCIAO and intensity 
M. de Juan Ovelar et al.: Imaging diagnostics for transitional discs

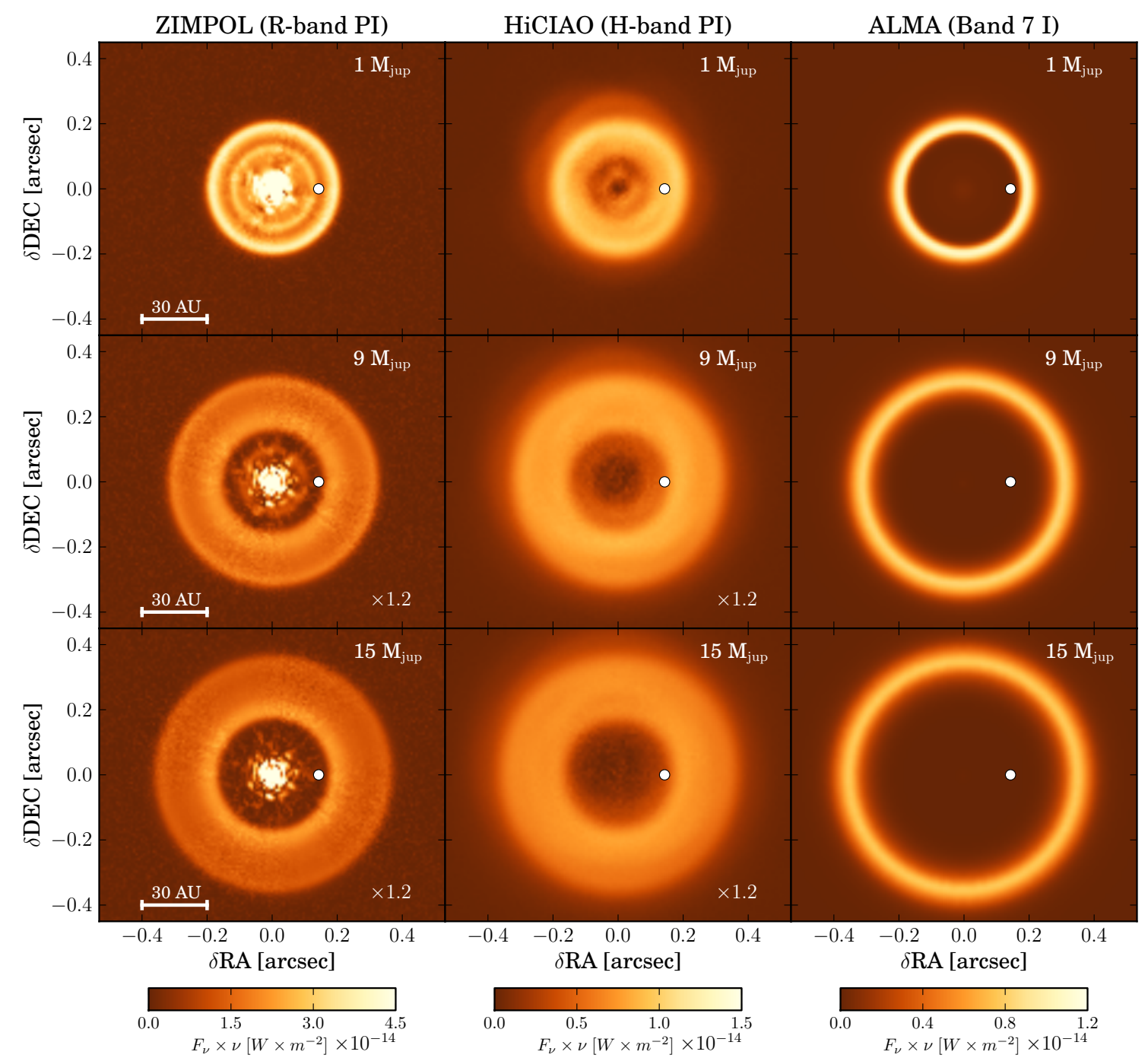

Fig. 2. Simulated images of the disc-planet models used in this study for the case of an embedded planet orbiting at 20 AU (white dot). Left to right columns correspond to polarised-intensity (PI) ZIMPOL images in the $R$-band $(0.65 \mu \mathrm{m})$, PI HiCIAO images in $H$-band $(1.6 \mu \mathrm{m})$ and intensity $(I)$ ALMA images at $850 \mu \mathrm{m}$. Top, middle and bottom rows show images obtained for planet masses of $M_{\mathrm{p}}=[1,9,15] M_{\text {Jup }}$ respectively. All images in the same band (column) share the colour scale, although the cases of 9 and $15 M_{\text {Jup }}$ in $R$ and $H$ polarised-intensity have been multiplied by a small factor to enhance the contrast. See Sect. 2 for details on how the models/images were generated.

images with ALMA, a gap of different sizes is also detected in all cases. However, these instruments do not detect any inner disc structure. Note that we did not use a coronagraph in our simulations to get to the smallest possible working angles. Published observations executed with HiCIAO often do use a coronagraph, masking what amounts to the inner disc in our simulations (see Sect. 4 below for details).

Figure 3 shows the computed intensity and polarisedintensity radial profiles of the full resolution and (instrument) limited resolution images for all mass cases studied and for a position of the planet of $R_{\mathrm{p}}=20 \mathrm{AU}$. We show profiles for intensity in $R(0.65 \mu \mathrm{m}), H(1.6 \mu \mathrm{m})$, and $850 \mu \mathrm{m}$ bands (solid lines) and polarised-intensity in $R(0.65 \mu \mathrm{m})$ and $H(1.6 \mu \mathrm{m})$ bands (dashed lines). Top to bottom panels show the cases of a planet mass of $M_{\mathrm{p}}=[1,9,15] M_{\mathrm{Jup}}$, respectively. Full resolution panels on the left, therefore show the theoretical emission and scattering pattern of the disc for each planet mass case, while the panels on the right (radial profiles of the simulated observations) show what would be imaged with the considered instruments (corresponding to the images in Fig. 2).

\subsection{Disc with a $1 M_{\text {Jup }}$ planet}

The top row of Fig. 2 shows the simulated observations with ZIMPOL, HiCIAO, and ALMA for the case of $M_{\mathrm{p}}=1 M_{\text {Jup }}$ at $R_{\mathrm{p}}=20 \mathrm{AU}$. Both polarimetric images obtained with ZIMPOL in $R$-band and HiCIAO in $H$-band detect scattered flux in the $R<30 \mathrm{AU}\left(\sim 0.2^{\prime \prime}\right)$ region that appears empty to ALMA at $850 \mu \mathrm{m}$. However, due to its high spatial resolution, ZIMPOL resolves a narrow and shallow gap at the position of the planet that is not detected by HiCIAO.

The top left panel in Fig. 3 shows the radial profiles of the full resolution images for this planet mass case (i.e., before applying the instrument simulators). In all these wavelengths, the morphology of the profiles can be divided into inner, $r<30 \mathrm{AU}$, and outer, $r>30 \mathrm{AU}$, regions. Visible and NIR wavelengths show a sharp decrease in the scattered and emitted flux at this position ( $r \sim 30 \mathrm{AU}$ ), where the emission at $850 \mu \mathrm{m}$ presents a narrow ( $\sim 10 \mathrm{AU}$ ) peak. In the inner $30 \mathrm{AU}$, the scattered flux in both $R$ and $H$ bands and the emission in $R$ show the narrow depletion of 


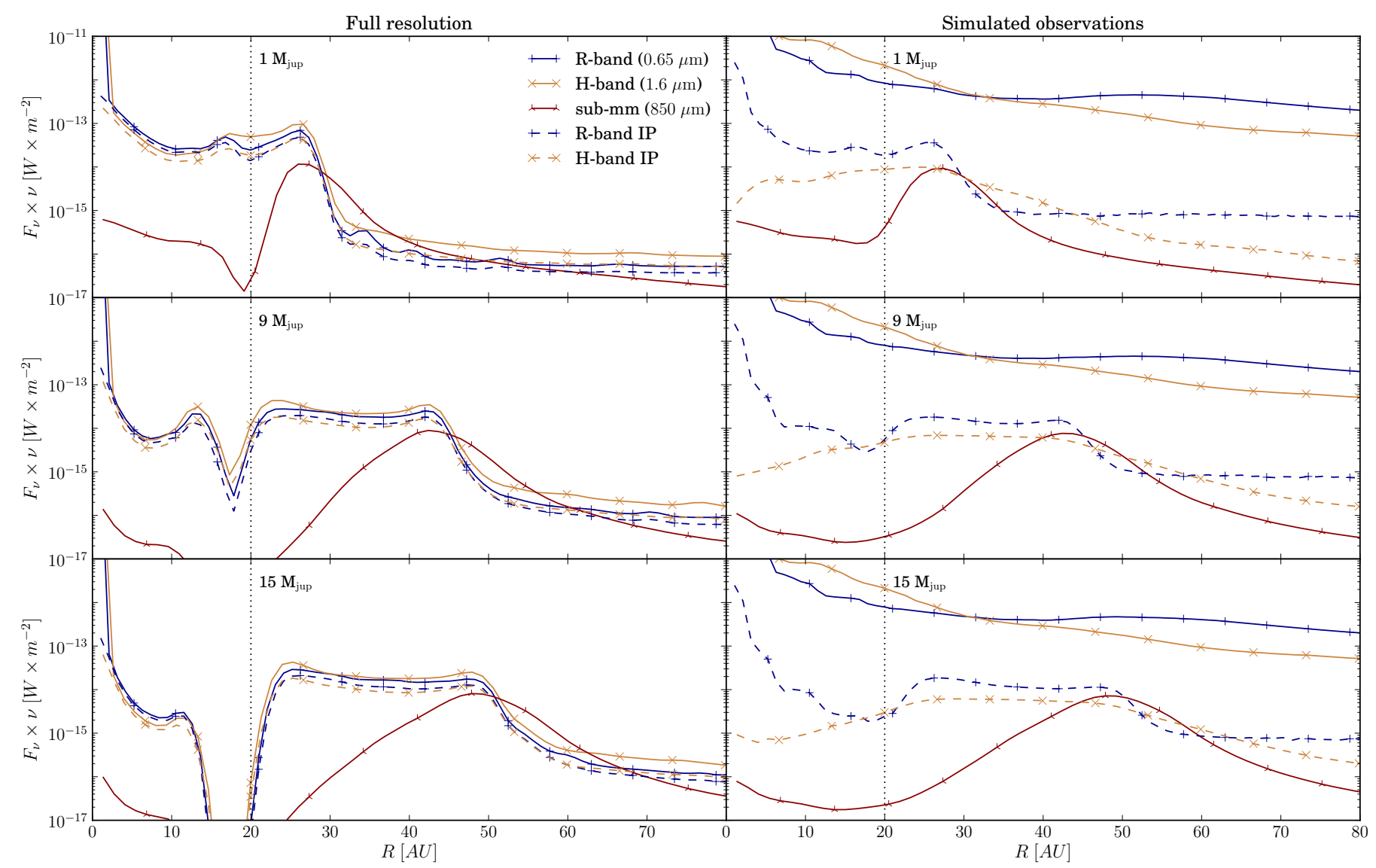

Fig. 3. Radial profiles of the theoretical images (left panels) and simulated observations (right panels) of a 3 Myr disc with a planet of mass $M_{\mathrm{p}}=[1,9,15] M_{\text {Jup }}$ from upper to lower panels in the $R(0.65 \mu \mathrm{m}), H(1.6 \mu \mathrm{m})$, and $850 \mu \mathrm{m}$ bands. Solid lines correspond to intensity $(I)$ profiles while dashed lines correspond to polarised-intensity (PI). The vertical dotted black line indicates the radial position of the planet at 20 AU.

a factor of $\sim 4$ at the position of the planet. The $850 \mu \mathrm{m}$ emission profile is strongly depleted in this region.

The top right panel in Fig. 3 shows the radial profiles of the simulated observations. Emission profiles at visible and NIR wavelengths ( $R$ and $H$ bands) are completely dominated by the flux of the central star; thus, the structure of the disc seen in the full resolution profiles is lost in the convolution with the PSF of the instrument. Images obtained with ALMA are immune to this effect, because the star is too faint at these wavelengths. The high resolution of the instrument also allows for the detection of the ring at about $30 \mathrm{AU}$.

Polarimetric observations in $R$ and $H$ efficiently remove the stellar emission from the image and are able to show the inner edge of the outer disc. However, only ZIMPOL images in $R$-band show the local depletion at the position of the planet because of the high resolution of the instrument.

These images and radial profiles agree with what is expected from the results of Pinilla et al. (2012a). The presence of the planet triggers the spatial separation of the different dust grain sizes (see bottom panels of Fig. 1). Big grains $(\sim 1 \mathrm{~mm})$ are trapped in the pressure maximum at about $\sim 30 \mathrm{AU}$, which is further out from the planet, and generate the emission detected by ALMA (i.e., ring at $\sim 30 \mathrm{AU})$. Small $(\sim 1 \mu \mathrm{m})$ grains are allowed in the radii closer to the star $(R<30 \mathrm{AU})$, and they are efficient scatterers at shorter wavelengths, which causes them to show up in the polarised-intensity images.

\subsection{Variation with planet mass}

For higher planet masses, the spatial separation of dust grain sizes becomes stronger (see middle and right bottom panels of Fig. 1). The 9 and $15 M_{\text {Jup }}$ planet mass cases are shown in the middle and lower panels of Figs. 2 and 3.

In both cases, the full resolution radial profiles show that both the outer sharp edge of the ring in visible and NIR wavelengths and the narrow emission ring at $850 \mu \mathrm{m}$ are now located at $\sim 50 \mathrm{AU}$. The decrease in flux at the position of the planet becomes larger than one order of magnitude at all wavelengths. The gap becomes deeper and wider in all bands, although this effect is stronger for the sub-mm wavelengths, indicating that, indeed, big grains of dust are more subject to the depletion generated by the planet at these radii.

The loss of structure in $R$ - and $H$-intensity images due to the convolution with the instrumental PSF is also present in these cases (blue and yellow solid lines in middle and lower right panels of Fig. 3). Again, ALMA is able to detect the overall shape of the corresponding profile in the theoretical images, showing a ring at about $r \sim 50 \mathrm{AU}$, where the maximum of the theoretical profiles is placed.

The polarimetric images are also able to detect the structure in the $R<50 \mathrm{AU}$ inner radii in these two planet mass cases. The $H$-band PI images with HiCIAO show an extended ring of scattered flux from $R \sim 10 \mathrm{AU}$ to $R \sim 50 \mathrm{AU}$ and from $R \sim$ $20 \mathrm{AU}$ to $R \sim 50 \mathrm{AU}$ for the intermediate and high planet mass cases, respectively. 
M. de Juan Ovelar et al.: Imaging diagnostics for transitional discs

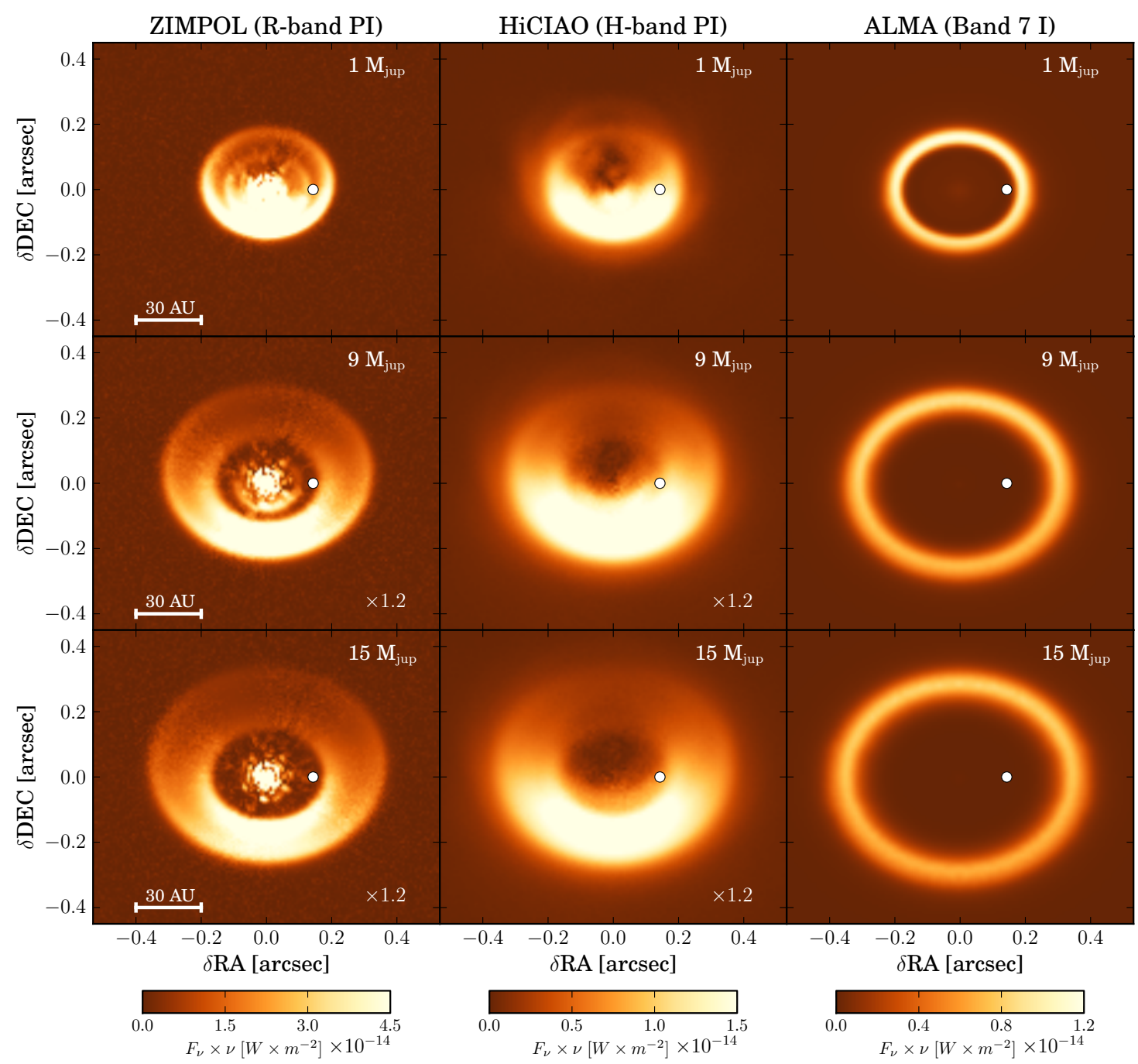

Fig. 4. Simulated images of the same discs as the ones shown in Fig. 2, now with an inclination of $i=35^{\circ}$ (with respect to pole-on). Again columns from left to right show ZIMPOL polarised-intensity in $R$-band $(0.65 \mu \mathrm{m})$, HiCIAO polarised intensity in $H$-band $(1.6 \mu \mathrm{m})$ and ALMA Band 7 $(850 \mu \mathrm{m})$ intensity images, respectively. Near and far sides of the disc correspond to lower and upper parts of the images, respectively.

The ZIMPOL images in $R$-band are particularly interesting in these cases. For the $9 M_{\text {Jup }}$ planet case, the simulated observation profile (dashed blue line in middle-right panel of Fig. 3) shows an inner ring that extends from $R \sim 5 \mathrm{AU}$ to $R \sim 15 \mathrm{AU}$ that is not present in the more massive $15 M_{\text {Jup }}$ case. The presence of this inner ring is a very interesting feature that differentiates between companion masses above or below the deuteriumburning limit of $\sim 13 M_{\text {Jup }}{ }^{1}$, which is often used as a dividing line between planets and brown dwarf companions. The full resolution radial profiles of the $H$ and $R$ images in these two cases (blue and yellow lines in middle and bottom left panels of Fig. 3) show that the scattered flux from the inner $\leq 17$ AU radii in the $9 M_{\text {Jup }}$ case is about an order of magnitude higher than that of the $15 M_{\text {Jup }}$ planet in that same region. Unfortunately, HiCIAO is not able to resolve it in the $H$-band, and this radial region in ZIMPOL images is dominated by speckles due to the proximity to the star. Therefore, its detection is not reliable enough, although the feature is resolved.

\footnotetext{
1 The deuterium-burning limit can range between 11 and $16 M_{\text {Jup }}$ depending on the metallicity (Spiegel et al. 2011).
}

\subsection{Effect of the disc inclination}

We have so far considered the case of a disc with a $i=0^{\circ}$ inclination (i.e., pole-on), but the emission and scattering images of inclined discs can differ considerably from this case. Figure 4 shows the same disc-planet cases presented in Fig. 2 (i.e., $M_{\mathrm{p}}=[1,9,15] M_{\mathrm{Jup}}$ at $R_{\mathrm{p}}=20 \mathrm{AU}$ ) with an inclination of $i=35^{\circ}$ (angle measured from pole-on). In general, the structural features (i.e., gaps and rings) remain the same, while the brightness patterns become asymmetrical. The polarised-intensity images show clearly the effect of forward scattering, which makes the near side of the disc appear brighter than the far side. This effect is caused by dust particles, which, depending on their size with respect to the incoming wavelength, can scatter light differently in different directions. In particular, grains with sizes $2 \pi a>\lambda$ are very strong forward scatterers, which means that the population of $a>1 \mu \mathrm{m}$ dust grains shows this effect in observations with ZIMPOL and HiCIAO PI at $\lambda \leq 1.6 \mu \mathrm{m}$. The difference in brightness between both sides can be used as an estimator of the size of dust particles in the disc, although one needs to be careful and appropriately account for the effects of dust particle shape and structure (Mulders et al. 2013). 
In the transitional discs we model in this study, the effect of the forward scattering turns out to be even more useful since it makes possible to detect the dust particles in the inner regions $\left(R<R_{\mathrm{p}}\right)$ of the disc more clearly. Indeed, the ZIMPOL images of the $35^{\circ}$ inclined discs for the $M_{\mathrm{p}}=9,15 M_{\text {Jup }}$ cases (middle and bottom images in the first column of Fig. 4) are now clearly distinguishable. The former shows scattering from the dust particles in the inner region of the disc, while the latter does not because the depletion of particles is much higher in this case (see Sect. 4 below for details).

The ALMA simulations show the opposite behaviour in the brightness pattern, where the far side appears brighter than the near side. This is simply because we are observing the emission of warmer dust in the far side and colder dust in the near side. In other words, we are looking directly at the wall of $1 \mathrm{~mm}$ particles illuminated by the star in the far side, while we are seeing the cold dust at the back of the disc in the near side.

\subsection{Variation with planet position}

As the planet orbits further away from the star, the basic inner disc-gap-outer disc morphology of the disc remains the same for all planet mass cases re-scaled to the position of the planet. Figure 5 shows ZIMPOL images in the $R$-band $(0.65 \mu \mathrm{m})$ for all three cases of planet mass studied before, now at planet orbit radii of $R_{\mathrm{p}}=[20,40,60] \mathrm{AU}$. The white dot again indicates the position of the planet. Overplotted, the contour black lines show the emission predicted for ALMA at $850 \mu \mathrm{m}$.

The images show how the inner disc becomes more extended for larger orbit radii in all planet mass cases. Also, due to the larger distance between the dust and the star, the scattering weakens and the ZIMPOL images become fainter. This effect also increases with the mass of the planet in the outer part of the disc. The radius of the ring detectable by ALMA (black contour lines), which traces the particle trap, increases in general with planet mass and separation. It is important to note how for the case of $M_{\mathrm{p}}=1 M_{\text {Jup }}$, the gap in the ZIMPOL images remains narrow almost exactly tracing the orbit of the planet at orbit radii of 20 and $40 \mathrm{AU}$. In the case of $R_{\mathrm{p}}=60 \mathrm{AU}$ the inner disc becomes too faint to distinguish the outer edge.

\subsection{Q-band $(20 \mu \mathrm{m})$ measurements}

Mid-infrared (MIR) emission measurements generally trace emission of warm dust grains of about $\sim 10 \mu \mathrm{m}$ in size. The presence of a circumstellar disc therefore broadens the footprint of the emission at these wavelengths and can cause the FWHM of the intensity profile to be larger than that of a point source. When a disc features an inner gap, the inner edge of the outer disc, or the wall, is exposed to the radiation from the central star and its temperature increases, which increases the MIR emission from that radial position. The PSF of imaging instruments that observe at these wavelengths is too large to resolve inner gaps (see Table 2), but the FWHM of the intensity profile can be used, with other diagnostics such as SED modelling, to estimate the position of the wall if a wall is present (Maaskant et al. 2013).

Figure 6 shows the variation in the intensity profile of our simulated observations at $20 \mu \mathrm{m}$ for all masses and positions of the planets explored in this study. The upper-row panels show variation with position for a fixed mass, while lower panels show variation with mass for a fixed position. In all panels, different colors correspond to different planet masses, while different line styles correspond to different planet positions. The profile of the calibrator star is shown as a striped black line for reference.

The generally accepted idea is that we expect more emission at these wavelengths when the wall is more exposed and closer to the star. In this sense, we can expect lower values for the FWHM when the semi-major axis of the orbit is larger for a fixed mass of the planet. On the other hand a heavier planet depleting more mass inside the gap leaves the wall more exposed to the radiation and should therefore correspond to larger values of the FWHM for a fixed planet separation. However, one should be careful when making these correlations, because the warm dust in the inner disc (if present) also has a contribution that is dominant in some cases, as our results show.

Indeed, we see in Fig. 6 that the exposure effect dominates when the position of the wall is relatively close to the star, which holds for all planet mass cases with semi-major axis at $20 \mathrm{AU}$ (lower left panel). More massive planets, which deplete more mass from the inner $\left(R \leq R_{\mathrm{p}}\right)$ radii, yield broader intensity profiles. However, the effect of the emission from the inner disc becomes increasingly important as the radial position of the planet increases. At $R_{\mathrm{p}}=40 \mathrm{AU}$ (lower middle panel), a $9 M_{\text {Jup }}$ planet broadens the intensity profile more than a $15 M_{\text {Jup }}$ planet. Since the wall is situated at roughly the same distance from the star (see images of Fig. 5), this can only be attributed to the emission of a more massive (i.e., less depleted) inner disc. We can see the effect as well in the upper panels of Fig. 6 .

At $R_{\mathrm{p}}=60 \mathrm{AU}$ (lower right panel), no measurable increase in the FWHM of the intensity profile is found for any planet mass. For all planet masses at this distance, the inner disc is too depleted and the wall is too far away from the star (i.e., too cold) to contribute to the $20 \mu \mathrm{m}$ emission.

For completeness, Fig. 9 of Appendix A shows the SEDs we obtained for all models presented in this study.

\section{Discussion}

As explained in Pinilla et al. (2012a), the presence of a massive planet affects the gas in the disc generating pressure gradients in the pressure distribution, which would otherwise decrease monotonously with increasing separation. If the disc is not perturbed by a planet, the negative gradient of the radial gas pressure makes the gas rotate with sub-Keplerian velocities while the dust moves with near-Keplerian velocities. This difference generates a drag force that causes the dust to lose momentum and spiral inwards (Weidenschilling 1977; Brauer et al. 2008). However, if a perturbation is present in the gas, whenever the pressure gradient is positive, the gas velocity grows until it becomes Keplerian in a pressure maximum. This counteracts the inward radial drift and causes the dust particles to accumulate in that position (Klahr \& Henning 1997; Fromang \& Nelson 2005; Brauer et al. 2008; Johansen et al. 2009; Pinilla et al. 2012b). What is interesting for our study is that the size of the particles that feel the trapping/filtering strongly depends on the strength of the pressure gradient (Pinilla et al. 2012a), which depends mainly on the characteristics of the planet (i.e., its mass) and the turbulence parameter $\alpha$. In general, the bigger a particle, the less susceptible it is to drag force, and the more easily it is trapped in the pressure maximum. Small particles, on the other hand, are able to filter through the pressure maximum and populate the inner regions (Rice et al. 2006).

As a consequence of these physical processes, the dust in the disc under the presence of a planet generally behaves as follows. Driven by the depletion of gas in radii close to the planet's orbit, 
M. de Juan Ovelar et al.: Imaging diagnostics for transitional discs

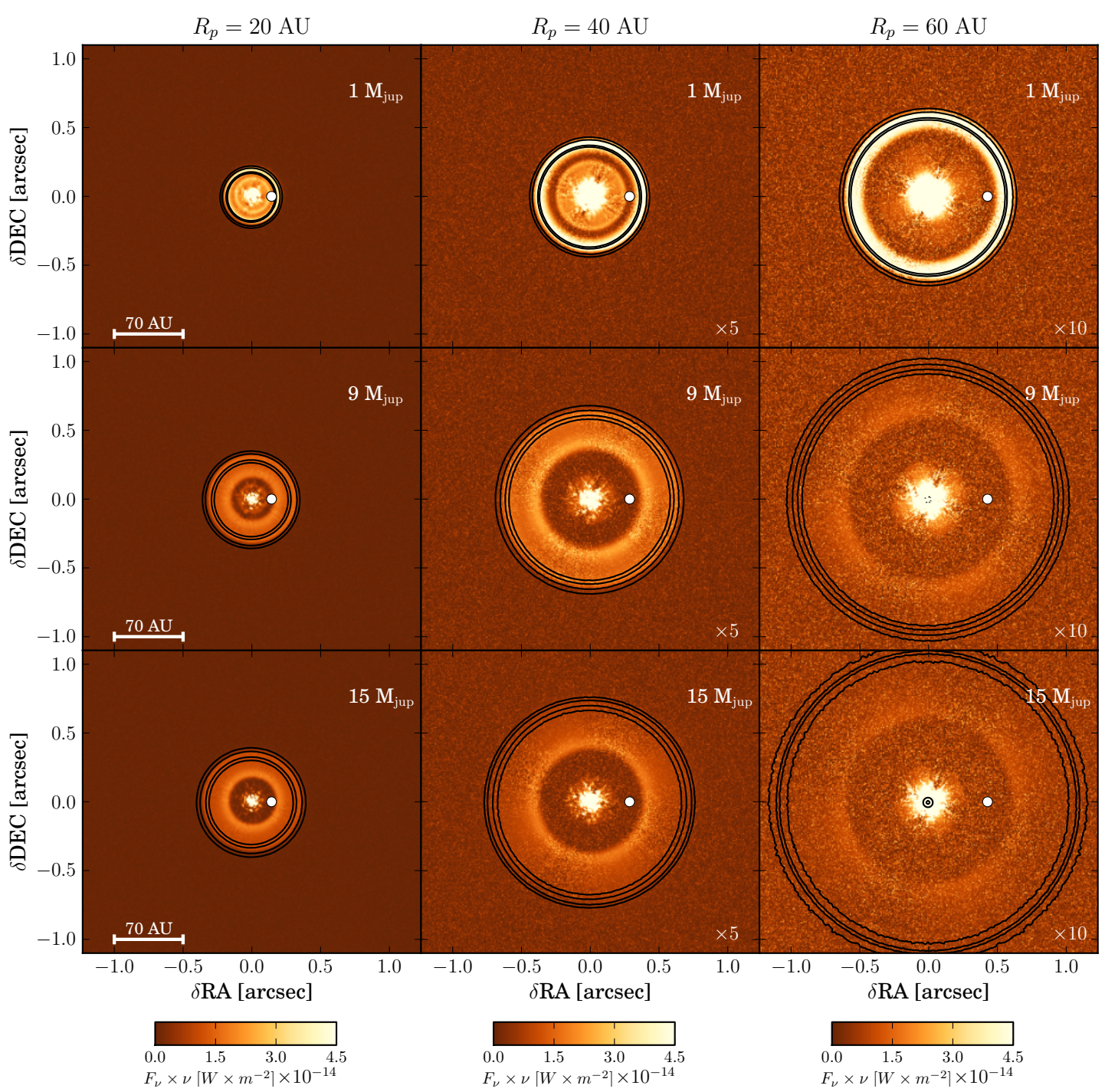

Fig. 5. Simulated ZIMPOL images of the different mass and orbit radius cases studied. Upper to bottom rows correspond to a $3 \mathrm{Myr}$ disc model with a planet of masses $M_{\mathrm{p}}=[1,9,15] M_{\mathrm{Jup}}$, respectively. Left to right columns show the different planet orbit cases, $R_{\mathrm{p}}=[20,40,60] \mathrm{AU}$, respectively. The white dot indicates the position of the planet in each image and the black contour lines correspond to the intensity ring detected by ALMA at $850 \mu \mathrm{m}$.

the dust distribution undergoes a depletion that affects different dust grain sizes in a different way. Big $(\sim 1 \mathrm{~mm})$ dust grains are depleted more easily and pushed to radii where the pressure is maximum, which is further away form the planet. Small $(\sim 1 \mu \mathrm{m})$ grains are able to remain at radii close to the planet, escape the trap generated by the pressure bump, and flow into inner regions due to the radial drift. As the planet becomes more massive, the depletion becomes stronger for all grain sizes, although the radial separation of different grain sizes remains. Figure 7 shows the surface density profiles of gas and different particle size bins in the planet mass cases studied and when the planet orbit radius is $R_{\mathrm{p}}=20 \mathrm{AU}$. The solid blue line represents the surface density of very small particles $\left(a=\left[10^{-4}-10^{-3}\right] \mathrm{cm}\right)$, while green, yellow, and red represent particles of increasing size up to a maximum of $a=1 \mathrm{~cm}$. Although the simulations consider particles sizes up to $2 \mathrm{~m}$, the contribution of particles larger than $1 \mathrm{~cm}$ to the emitted and scattered light is negligible, so we did not include them in this plot for the sake of simplicity. According to the results presented in this study, the morphology of the dust distribution does not change with the planet's orbital radius but scales with it.

\subsection{Visible and near-infrared polarimetric images vs. sub-mm observations}

Since observations at different wavelengths generally trace different particle sizes, this spatial differentiation of the size distribution of dust in the disc opens the possibility of constraining planet parameters such as the mass and separation through multi-wavelength images of the emitted and/or scattered light from the disc. The images and radial profiles shown in Sect. 3 illustrate how the different observations trace different parts of this distribution. In general, ZIMPOL and HiCIAO polarisedintensity images trace particles of about $1 \rightarrow 10 \mu \mathrm{m}$ (blue solid line in Fig. 7), because these particles are very efficient scatterers at these wavelengths, while bigger grains are not. Images taken with ALMA Band $7(\lambda \sim 850 \mu \mathrm{m})$ are very sensitive to emission from grains of about $1 \mathrm{~mm}$, and they, therefore, highlight the ring of big particles trapped in the pressure maximum, while the inner regions, which are heavily depleted of these grains, appear empty.

The ZIMPOL and HiCIAO images are different mainly because of resolution. Observing at shorter wavelengths, ZIMPOL is able to resolve the scattering due to the small grains in the 


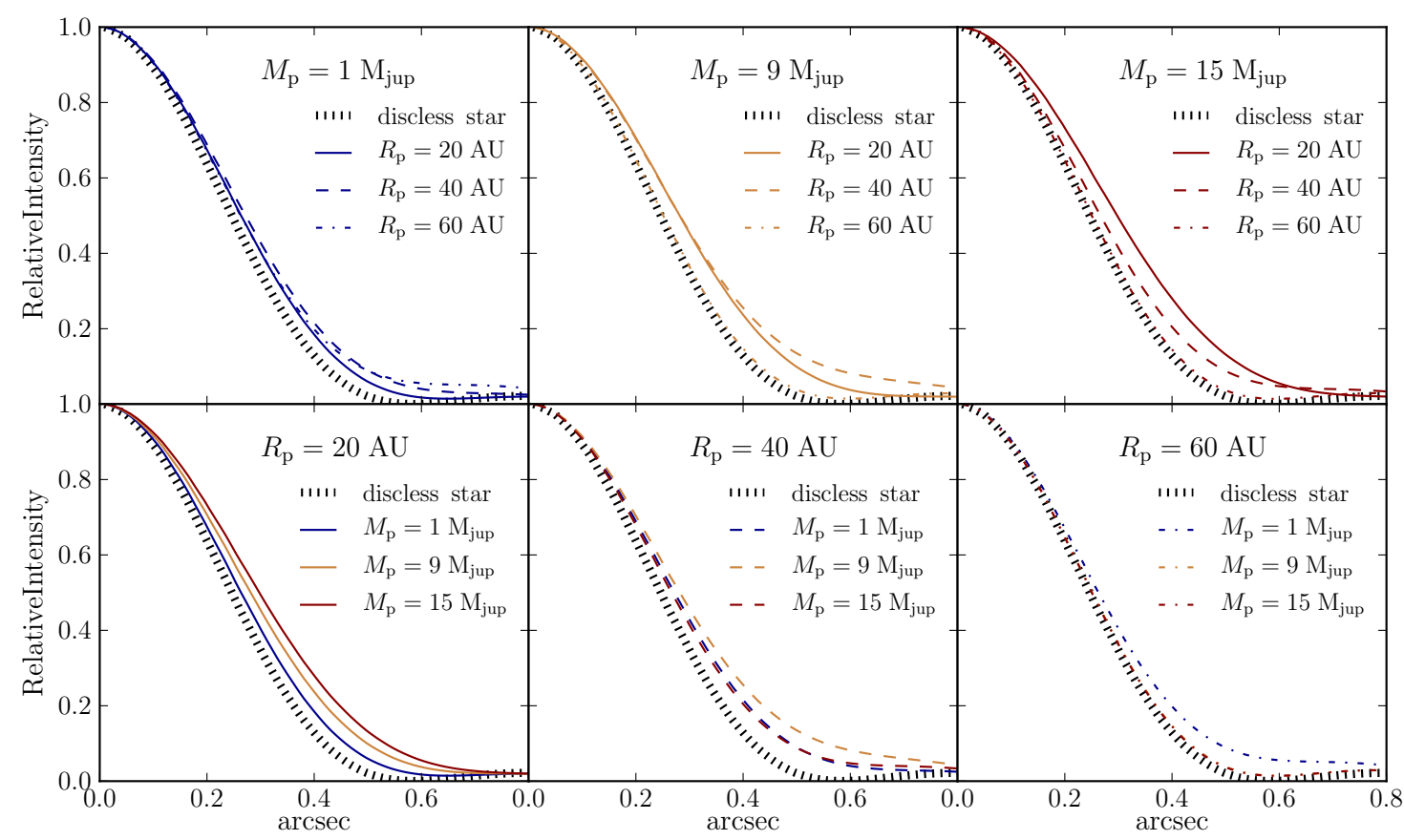

Fig. 6. Synthetic $20 \mu \mathrm{m}$ ( $Q$-band) measurements for the different masses and planet positions considered. Upper row: variation in the PSF profile with planet position for fixed planet mass. Lower row: variation in the PSF profile with planet mass for a fixed planet position. In all panels, the different colors indicate different planet masses (blue, yellow, and red for $M_{\mathrm{p}}=[1,9,15] M_{\text {Jup }}$, respectively) while different line styles correspond to different planet positions (solid, dashed and dash-dotted for $R_{\mathrm{p}}=[20,40,60] \mathrm{AU}$, respectively). The black striped line shows the PSF corresponding to a discless (calibrator) star for reference.

inner region of the disc (down to the separation of the planet), while HiCIAO is only able to detect the outer part. It is important to note that our simulated HiCIAO images do not include the remnants of the star in the center of the image, which are visible in ZIMPOL images. This is because HiCIAO observations are simulated by convolving polarimetric full resolution images that come out of MCMax with the measured PSF of the instrument at $H$-band. Here, we assume that the star is totally unpolarised and that polarimetry is perfectly done, which suppresses the starlight completely. ZIMPOL images are simulated more realistically using the ZIMPOL simulator, which includes the speckle pattern that would remain in the observations due to imperfect polarimetry.

The presence of an inner disc in ZIMPOL images could directly differentiate between a companion with mass below and above the deuterium-burning limit, since in the latter the depletion of small grains is so strong in these regions that the scattered flux is not detected. Moreover, a close look at the images of Fig. 5 suggests a relation between the radial positions of the wall detected in ZIMPOL polarimetric images and the ALMA sub$\mathrm{mm}$ emission peak at $850 \mu \mathrm{m}$ (black contours) which varies with planet mass but remains approximately constant with planet separation. To investigate this more, we compute the ratio of these features for each planet mass and separation case studied.

Figure 8 shows the ratios versus planet mass for planet orbit radii of $R_{\mathrm{p}}=[20,40,60] \mathrm{AU}$. Using the radial profiles of the images, we define the position of the wall as the radial position of half the flux difference between the minimum flux at the bottom of the gap and the maximum flux at the wall (e.g., $23 \mathrm{AU}$ and $27 \mathrm{AU}$ for minimum and maximum flux positions, respectively, in the case of $1 M_{\mathrm{Jup}}$ at $20 \mathrm{AU}$. See the dashed blue line of upper right panel in Fig. 3). The upper and lower errors of the position are obtained by propagating the error on the determination of the mid-flux point and the peak of the emission in ZIMPOL and ALMA profiles, respectively. Whenever those errors are lower than the corresponding resolution element (4 AU and $2 \mathrm{AU}$ for ZIMPOL and ALMA, respectively), the resolution element is used instead. The position of the points of the same planet mass in the figure are slightly offset to facilitate their distinction.

The ratios for each planet's orbit radius are best fitted with a power law,

$f\left(M_{\mathrm{p}}\right)=c \cdot\left(\frac{M_{\mathrm{p}}}{M_{\mathrm{Jup}}}\right)^{\gamma}$

with $c \sim 0.85$ and $\gamma \sim[-0.22,-0.18,-0.16]$ for $R_{\mathrm{p}}=$ $[20,40,60]$ AU orbit radii, respectively.

Within the framework of our models, this figure serves as a mass estimator for the planet.

\subsection{Mid-infrared vs. near-infrared polarimetric observations}

Interestingly, recent observational work also presented differences between MIR and polarimetric observations. In a recent paper, Maaskant et al. (2013) presented the case of HD 97048 (a Herbig Be/Ae star with a group I flared disc), where $24.5 \mu \mathrm{m}$ T-ReCS (Gemini South) measurements agreed with a model of the disc that features a gap of $\sim 30 \mathrm{AU}$ (i.e., a wall positioned at $30 \mathrm{AU})$. The reason why this is interesting is that this was the first study that suggested the presence of a gap in this disc, despite the fact that this target has been observed in multiple occasions at different wavelengths (van Boekel et al. 2004; Lagage et al. 2006; Acke \& van den Ancker 2006; Doucet et al. 2007; Doering et al. 2007; Martin-Zaïdi et al. 2007; van der Plas et al. 2009; Müller et al. 2011; Quanz et al. 2012). The closest-in observation, probing radial distances from $\sim 16-160 \mathrm{AU}$, was made by Quanz et al. (2012), who took VLT/NACO $H$ - and $K_{\mathrm{s}}$-band polarimetric images where no gap was detected.

According to the results presented in this paper, these apparently discrepant observations would be consistent with the 
M. de Juan Ovelar et al.: Imaging diagnostics for transitional discs

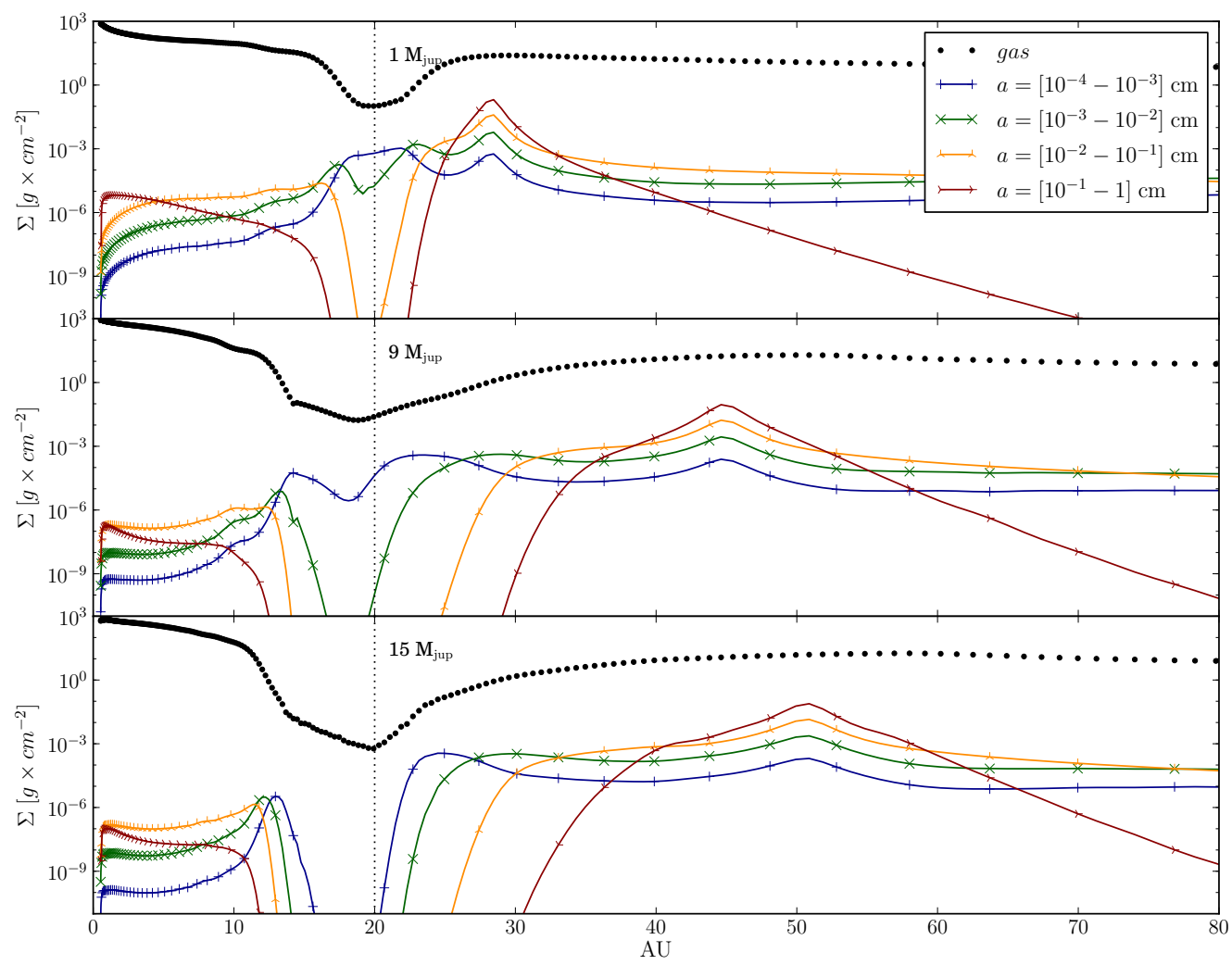

Fig. 7. Surface density profiles for gas and different dust particle size ranges for the three planet mass cases, i.e. $M_{\mathrm{p}}=[1,9,15] M_{\mathrm{Jup}}$. The vertical dotted line indicates the position of the planet at $20 \mathrm{AU}$.

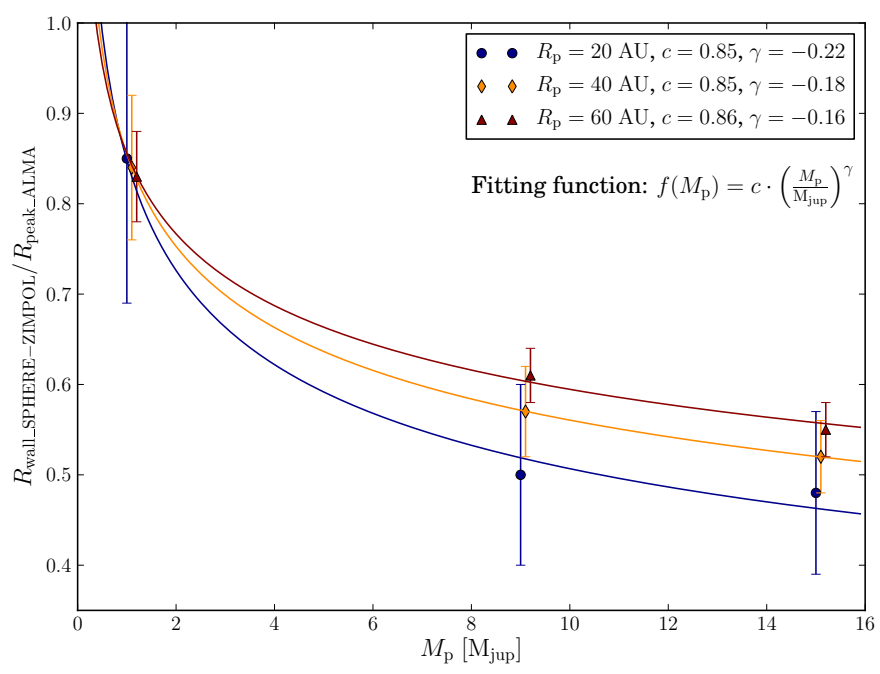

Fig. 8. SPHERE ZIMPOL $R$-band outer gap edge to ALMA Band 7 peak ratio versus planet mass for the three planet orbit radii studied $R_{\mathrm{p}}=[20,40,60] \mathrm{AU}$.

case of a disc hosting a planet of $1 M_{\text {Jup }}$ or $9 M_{\text {Jup }}$ at $20 \mathrm{AU}$. Indeed, the $H$-band Subaru/HiCIAO polarimetric images (similar to what would be observed with VLT/NACO) do not show a gap for these cases (second column upper panel in Fig. 2) other than the inner $<10$ AU gap, which can be attributed to the assumption of a perfectly suppressed central source (see Sect. 4.3 below). The $9 M_{\text {Jup }}$ case, however, shows a depletion in the inner $<20$ AU that would have been most likely detected by the Quanz et al. (2012) observations. If we discard the larger planet mass case based on this, the upper left panel of Fig. 6 shows how the PSF measured at $20 \mu \mathrm{m}$ would indeed appear broadened for the
$1 M_{\text {Jup }}$ case. Here, the cases of $1 M_{\text {Jup }}$ at $20 \mathrm{AU}, 1 M_{\text {Jup }}$ at $40 \mathrm{AU}$ and $15 M_{\text {Jup }}$ at $40 \mathrm{AU}$ (lower central panel) would all be consistent with the same broadening but the polarimetric $H$-band images do not show a gap or a depletion of dust in these regions, which discards the two latter options.

We note that the simulations presented here cannot be directly compared to either NACO or T-ReCS observations presented in those studies or used as predictions for future observations of this target, since the parameters of our star-disc system may differ considerably from those of HD 97048. Therefore, we do not claim that a $1 M_{\text {Jup }}$ planet at $20 \mathrm{AU}$ is indeed responsible for the observational diagnostics presented in those papers. We simply use this observational case as a proof of concept of our diagnostic method where polarimetric imaging at short wavelengths can be used to disentangle otherwise degenerate cases that would agree with measurements at larger wavelengths.

\subsection{Instrumental considerations}

Regarding instrumental performance and considering that the simulated images presented in this study for ZIMPOL and ALMA consider capabilities not available yet, it is clear that, whenever available, the upcoming ZIMPOL polarimeter will provide unprecedented spatial resolution for polarimetric images. This is a big advantage, since currently available polarimeters, such as Subaru/HiCIAO or VLT/NACO, do not have the power to probe the innermost regions of the disc and to accurately resolve the outer edge of the gap. According to the results of this study, these features are of extreme importance to properly constrain the mass and position of the planet. Although we are aware that our simulations have limitations (e.g., accretion of dust from the inner disc onto the star is not taken into account, and we only consider the presence of one planet) and cannot 
represent all possible disc-planet systems, they serve as a proof of concept for the power of using combined interferometric and polarimetric images to characterise these objects.

The results are in clear agreement with the differences observed between the SEEDS images obtained with HiCIAO and the interferometric results on the same targets. The gap shown by the ALMA images at $850 \mu \mathrm{m}$ is larger than the gap in HiCIAO polarised-intensity images at $1.6 \mu \mathrm{m}$ by a factor of $>2$ in radial extent for all planet mass cases. If the disc is one of the $1 M_{\mathrm{Jup}}$ type simulated here (or a less massive single planet), the gap in polarised-intensity could be too small to even be detected. Moreover, if the polarimetric images are taken using a coronagraph, as is the case for most of the SEEDS observations, the gap can be hidden. From the disc-planet systems considered in our simulations, observations with HiCIAO in the $H$-band using the coronagraph $\left(d_{\text {eff.coro. }} \sim 0.18^{\prime \prime}\right.$, Dong et al. 2012) would have missed all gaps generated by planets lighter than (and including) $9 M_{\text {Jup }}$. Although the basic effect has been shown in parametric models before, this is the first time that self-consistent physical models have been used to explain these observational discrepancies.

\section{Summary and conclusions}

We present simulated imaging observations of a protoplanetary disc with a planet of masses, $M_{\mathrm{p}}=[1,9,15] M_{\mathrm{Jup}}$ embedded in the disc and orbiting at $R_{\mathrm{p}}=[20,40,60] \mathrm{AU}$. We simulate intensity and polarised-intensity images in the visible, nearinfrared, mid-infrared, and sub-mm wavelengths with current and near-future ground-based imaging instruments with the aim of 1) testing whether the proposed models explain observed differences between multi-wavelength observations recently presented; 2) studying what different images can tell us about the dust distribution in the disc; and 3) finding the best imaging strategy to infer planet mass and position. To simulate the disc-planet systems, we use the models presented in Pinilla et al. (2012a) that combine 2D hydrodynamical and state-of-the-art dust evolution simulations to self-consistently compute the evolution of the dust and the gas in the system including radial drift, fragmentation, and coagulation processes (Masset 2000; Birnstiel et al. 2010).

To simulate the observations, we input the gas and dust radial surface density distribution obtained from the models after $3 \mathrm{Myr}$ of evolution into the the Monte-Carlo radiative transfer code MCMax to obtain the theoretical emission and scattered light images for the six planet masses and positions studied. We then process these theoretical images in different ways to simulate observations with the upcoming SPHERE ZIMPOL polarimeter in the $R$-band $(0.65 \mu \mathrm{m}$ intensity and polarisedintensity images), HiCIAO in the $H$-band $(1.6 \mu \mathrm{m}$ intensity and polarised-intensity images), VLT-VISIR in the $Q$-band $(20 \mu \mathrm{m})$, and ALMA $(850 \mu \mathrm{m})$ with its future complete capabilities.

We find that:

1. The trapping and filtering mechanisms triggered by the presence of a $>1 M_{\text {Jup }}$ planet in the disc lead to different radial dust distributions for different grain sizes. This causes observations at different wavelengths to show different structures. Particles with large sizes $(\sim 1 \mathrm{~mm})$ are trapped in the pressure maximum outside the planet's orbit, while smaller particles $(\sim 1 \mu \mathrm{m})$ are allowed to drift to the inner radii. The former show up in sub-mm emission measurements as a ring at the radial position of the pressure maximum, while the latter are detected in polarised scattering flux at inner radii.
The position of the pressure trap, the amount of particles trapped and filtered, and the particle size threshold of the filtering process depend strongly on the mass of the planet. This effect allows to constrain planet mass and separation by combining multi-wavelength observations.

2. The Pinilla et al. (2012a) models are able to reproduce the "missing cavities" problem presented by the SEEDS survey (Dong et al. 2012; $H$-band images in this study), where no gaps were found in polarimetric $H$-band images for targets known to exhibit gaps at $850 \mu \mathrm{m}$. Our simulated images with HiCIAO assume perfect polarimetry and a totally unpolarised star, which makes it just possible to detect a gap for all planet mass cases. In reality, speckle noise and, if used, the presence of a coronagraph would make the detection of the gap very unlikely.

3. Combination of sub-mm and polarimetric images in the visible wavelength range is the best imaging strategy to characterise the main features of the dust grain size distribution. An instrument like SPHERE-ZIMPOL in the $R$-band $(\lambda=0.65 \mu \mathrm{m})$ could differentiate between the three planet mass cases we study in polarised-intensity, particularly if the system is inclined. The high spatial resolution provided by the instrument allows us to resolve the different structures in the inner region ([10-20] AU), or lack thereof, that each mass generates, which is currently not possible using any other instruments.

4. Combination of near-infrared polarimetric images and midinfrared measurements can also yield constraints on mass and planet position, although less accurately than the combination of diagnostics proposed as the best in this study (visible polarimetric and sub-mm images). This is because the spatial resolution of polarimetric images at these wavelengths $(1.6 \mu \mathrm{m})$ is currently not enough to resolve the inner regions to the position of the planet. The warm dust in these regions also contributes to the FWHM measured at mid-infrared wavelengths. This causes some degeneracy in the models that would fit the measurements. Not having detailed spatial information in the polarimetric images for these regions (or even the gaps, which could be detected with SPHERE ZIMPOL) can make it difficult to disentangle the different planet mass and separation cases.

5. The results also agree with the discrepancy between $Q$-band and near-infrared polarimetric observations of Maaskant et al. (2013) and Quanz et al. (2012) respectively, where a gap of $\sim 30 \mathrm{AU}$ was found by modelling the $Q$-band emission (at $24.5 \mu \mathrm{m}$ ), while NACO polarimetric images in the $H$ - and $K_{\mathrm{s}}$-band did not show this feature.

Acknowledgements. The authors are grateful to Koen Maaskant, Gijs Mulders, and the "API cookie crew" for helpful and insightful discussions, comments, and suggestions during the course of the study. M.M. acknowledges funding from the EU FP7-2011 under Grant Agreement No. 284405. T.B. acknowledges support from NASA Origins of Solar Systems grant NNX12AJ04G.

\section{Appendix A: Spectral energy distributions}

Figure 9 shows the spectral energy distributions (SEDs) of the models used in this study. The format is the same as that of Fig. 6, where upper panels show SED variation with planet separation for a fixed planet mass and lower panels show variation with planet mass for a fixed planet separation.

The depletion of the SED in the range of $\lambda \approx[20-100] \mu \mathrm{m}$ increases systematically with both planet mass and separation. For longer wavelengths, the flux increases with planet separation 


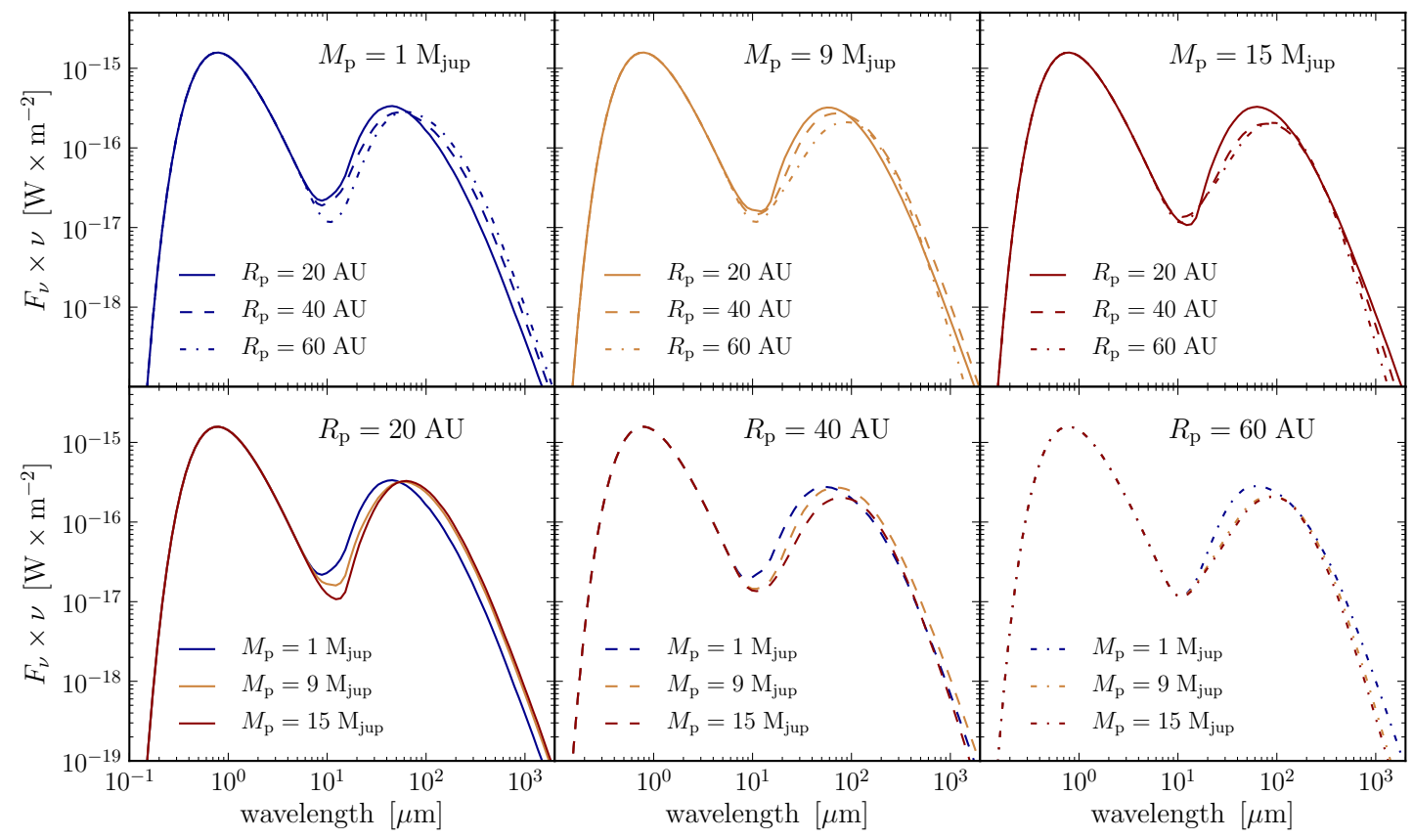

Fig. 9. Synthetic SEDs obtained for the different masses and planet positions studied. Upper row: variation in the SED with planet position for a fixed planet mass. Lower row: variation in the SED with a planet mass for a fixed planet position. In all panels, the different colors indicate different planet masses (blue, yellow and red for $M_{\mathrm{p}}=[1,9,15] M_{\mathrm{Jup}}$, respectively), while different line styles correspond to different planet positions (solid, dashed and dash-dotted for $R_{\mathrm{p}}=[20,40,60] \mathrm{AU}$, respectively).

for the lowest planet mass case in the far-infrared to millimetre regime (i.e. $\lambda \approx[100-1000] \mu \mathrm{m}$ ), whereas this relation seems to reverse as the mass increases. At the highest planet mass case, there is not significant variation with planet separation until $\lambda>$ $1 \mathrm{~mm}$.

For fixed planet separations, the variation in the SED with mass in this wavelength range is only significant for the closest value, $R_{\mathrm{p}}=20 \mathrm{AU}$ although the lightest planet in the case of $R_{\mathrm{p}}=60 \mathrm{AU}$ shows a slight excess in flux with respect to the $M_{\mathrm{p}}=[9,15] M_{\text {Jup }}$.

\section{References}

Acke, B., \& van den Ancker, M. E. 2006, A\&A, 449, 267

Andrews, S. M., Wilner, D. J., Espaillat, C., et al. 2011, ApJ, 732, 42

Ataiee, S., Pinilla, P., Zsom, A., et al. 2013, A\&A, 553, L3

Begemann, B., Dorschner, J., Henning, T., Mutschke, H., \& Thamm, E. 1994, ApJ, 423, L71

Beuzit, J.-L., Feldt, M., Dohlen, K., et al. 2006, The Messenger, 125, 29

Birnstiel, T., Dullemond, C. P., \& Brauer, F. 2010, A\&A, 513, A79

Brandt, T. D., McElwain, M. W., Turner, E. L., et al. 2013, ApJ, 764, 183

Brauer, F., Dullemond, C. P., \& Henning, T. 2008, A\&A, 480, 859

Calvet, N., D'Alessio, P., Watson, D. M., et al. 2005, ApJ, 630, L185

Canovas, H., Min, M., Jeffers, S. V., Rodenhuis, M., \& Keller, C. U. 2012, A\&A, 543, A70

de Vries, B. L., Acke, B., Blommaert, J. A. D. L., et al. 2012, Nature, 490, 74

Doering, R. L., Meixner, M., Holfeltz, S. T., et al. 2007, AJ, 133, 2122

Dong, R., Rafikov, R., Zhu, Z., et al. 2012, ApJ, 750, 161

Dorschner, J., Begemann, B., Henning, T., Jaeger, C., \& Mutschke, H. 1995, A\&A, 300, 503

Doucet, C., Habart, E., Pantin, E., et al. 2007, A\&A, 470, 625

Espaillat, C., Calvet, N., D’Alessio, P., et al. 2007, ApJ, 670, L135

Espaillat, C., D’Alessio, P., Hernández, J., et al. 2010, ApJ, 717, 441

Fromang, S., \& Nelson, R. P. 2005, MNRAS, 364, L81

Gisler, D., Schmid, H. M., Thalmann, C., et al. 2004, Proc. SPIE, 5492, 463

Henning, T., \& Stognienko, R. 1996, A\&A, 311, 291

Honda, M., Maaskant, K., Okamoto, Y. K., et al. 2012, ApJ, 752, 143

Jeffers, S. V., Min, M., Waters, L. B. F. M., et al. 2012, A\&A, 539, A56
Johansen, A., Youdin, A., \& Klahr, H. 2009, ApJ, 697, 1269

Klahr, H. H., \& Henning, T. 1997, Icarus, 128, 213

Lagage, P. O., Doucet, C., Pantin, E., et al. 2006, Science, 314, 621

Lombaert, R., de Vries, B. L., de Koter, A., et al. 2012, A\&A, 544, L18

Maaskant, K. M., Honda, M., Waters, L. B. F. M., et al. 2013, A\&A, 555, A64

Martin-Zaïdi, C., Lagage, P. O., Pantin, E., \& Habart, E. 2007, ApJ, 666, L117

Masset, F. 2000, A\&AS, 141, 165

Min, M., Dullemond, C. P., Dominik, C., Koter, A. D., \& Hovenier, J. W. 2009, A\&A, 497, 155

Min, M., Dullemond, C. P., Kama, M., \& Dominik, C. 2011, Icarus, 212, 416

Min, M., Canovas, H., Mulders, G. D., \& Keller, C. U. 2012, A\&A, 537, A75

Mulders, G. D., \& Dominik, C. 2012, A\&A, 539, A9

Mulders, G. D., Dominik, C., \& Min, M. 2010, A\&A, 512, A11

Mulders, G. D., Waters, L. B. F. M., Dominik, C., et al. 2011, A\&A, 531, A93

Mulders, G. D., Min, M., Dominik, C., Debes, J. H., \& Schneider, G. 2013, A\&A, 549, A112

Müller, A., Carmona, A., van den Ancker, M. E., et al. 2011, A\&A, 535, L3

Pinilla, P., Benisty, M., \& Birnstiel, T. 2012a, A\&A, 545, A81

Pinilla, P., Birnstiel, T., Ricci, L., et al. 2012b, A\&A, 538, A114

Preibisch, T., Ossenkopf, V., Yorke, H. W., \& Henning, T. 1993, A\&A, 279, 577

Quanz, S. P., Birkmann, S. M., Apai, D., Wolf, S., \& Henning, T. 2012, A\&A, 538, A92

Rice, W. K. M., Armitage, P. J., Wood, K., \& Lodato, G. 2006, MNRAS, 373, 1619

Roelfsema, R., Schmid, H. M., Pragt, J., et al. 2010, Proc. SPIE, 7735

Schmid, H. M., Beuzit, J. L., Mouillet, D., et al. 2010, in Proc. Conf. in the Spirit of Lyot, ed. Boccaletti

Spiegel, D. S., Burrows, A., \& Milsom, J. A. 2011, ApJ, 727, 57

Strom, K. M., Strom, S. E., Edwards, S., Cabrit, S., \& Skrutskie, M. F. 1989, AJ, 97, 1451

Stuik, R., Tinbergen, J., Joos, F., \& Schmid, H. M. 2005, Proc. SPIE, 343, 94

Tamura, M. 2009, in AIP Conf. Ser. 1158, eds. T. Usuda, M. Tamura, \& M. Ishii, 11

Thalmann, C., Schmid, H. M., Boccaletti, A., et al. 2008, Proc. SPIE, 7014, $70143 \mathrm{~F}$

van Boekel, R., Min, M., Leinert, C., et al. 2004, Nature, 432, 479

van der Plas, G., van den Ancker, M. E., Acke, B., et al. 2009, A\&A, 500, 1137

Weidenschilling, S. J. 1977, MNRAS, 180, 57

Williams, J. P., \& Cieza, L. A. 2011, ARA\&A, 49, 67

Zhu, Z., Nelson, R. P., Hartmann, L., Espaillat, C., \& Calvet, N. 2011, ApJ, 729, 47

Zhu, Z., Nelson, R. P., Dong, R., Espaillat, C., \& Hartmann, L. 2012, ApJ, 755, 6 